\title{
Characterizing the correlation between column density structure and magnetic fields
}

\author{
J. D. Soler ${ }^{1}$, P. Hennebelle ${ }^{2}$, P. G. Martin ${ }^{3}$, M. A. Miville-Deschenes ${ }^{4}$, \\ B. Netterfield ${ }^{1}$ and The BLASTpol Collaboration ${ }^{5}$ \\ ${ }^{1}$ Dept. of Astronomy and Astrophysics, University of Toronto, Toronto, ON M5S 3H4, Canada \\ email: soler@astro.utoronto.ca, ${ }^{2}$ Lab. de Radioastronomie, ENS et Observatoire de Paris, \\ 75231 Paris, France ${ }^{3}$ CITA, Toronto, ON M5S 3H8, Canada ${ }^{4}$ Institut d'Astrophysique \\ Spatiale, CNRS/Universit Paris-Sud 11, 91405 Orsay, France ${ }^{5}$ http://blastexperiment.info
}

\begin{abstract}
We present a statistical tool to characterize correlation between the column density structure and the polarized emission from a molecular cloud. This tool uses the gradient as an estimator of the directionality of the structure in order to systematically relate the orientation of filaments and cores to the orientation of the magnetic field inferred from the polarization vectors.
\end{abstract}

Keywords. ISM: magnetic fields, ISM: clouds, polarization, submillimeter

The importance of magnetic fields in the process of star formation is not yet determined (Crutcher, 2012). Recent studies try to relate the morphology of the magnetic field in molecular clouds to the processes which lead to the formation of filaments and subsequently to the formation of stars (e.g. Sugitani, et al. 2011 and Girart, et al. 2006). In this context, we introduce the Histograms of Oriented Gradients (HOGs), a statistical tool to quantitatively compare the orientation of structures with respect to the magnetic field $(\mathbf{B})$ inferred from dust polarization observations. We developed this tool by characterizing 3D data cubes and 2D maps from simulations of molecular clouds. The results of the analysis are histograms of the angles between $\mathbf{B}$ and the gradient of the density $(\nabla \rho)$ or column density $(\nabla \Sigma)$. We calculate the gradient using Gaussian derivative kernels to sample different scales in the map and make the measurement robust against noise. Additionally we segment the cubes or the maps by intervals of density and magnetic field intensity.

\section{Conclusion}

We applied HOGs to column density and polarization maps built from simulations, assuming perfect grain alignment. We find two distinct modes of orientation between $\mathbf{B}$ and filament direction correlated with the column density.

After producing simulated BLASTPol observations (Pascale, et al. 2008) of our polarization maps, we show this correlation should be measurable with BLASTPol. Potentially, this method can also be applied to the observations of other submillimeter instruments such as Planck, POL-2 and PILOT.

\section{References}

Crutcher, R. M., 2012, ARA\&A 50, 29C

Girart, J. M., Rao, R., \& Marrone, D. P., 2006, Science 313, 812G

Pascale, E., et al. 2008, ApJ 681, 400

Sugitani, E., et al. 2011, ApJ 734, 63S 